\title{
Dynamical Examinations of Vibrator Models, Describing Some Non Elastic Properties of Crystals
}

\author{
Vladimir Kh. Kozlovskiy \\ Scientific Society, Berlin, Germany \\ Email: Kozlovskiy.V@t-online.de
}

How to cite this paper: Kozlovskiy V.K. (2017) Dynamical Examinations of Vibrator Models, Describing Some Non Elastic Properties of Crystals. World Journal of Condensed Matter Physics, 7, 80-88. https://doi.org/10.4236/wicmp.2017.73007

Received: July 11, 2017

Accepted: August 21, 2017

Published: August 24, 2017

Copyright $\odot 2017$ by author and Scientific Research Publishing Inc. This work is licensed under the Creative Commons Attribution International License (CC BY 4.0).

http://creativecommons.org/licenses/by/4.0/

\section{c) (i) Open Access}

\begin{abstract}
On the base of a vibrator atomic model the mechanical and thermal properties of the object are analyzed. The potential energy of the vibrator is represented by means of positive term with coordinate deflection in second power and negative term with deflection in fourth power. With the use of dynamical procedure of calculation, which permits to calculate mean deflection and root mean square amplitude of vibrations, the dependence of applied force from mean amplitude and temperature is calculated. This dependence shows a maximum (or minimum, when the direction of force is reversed), the height of which diminishes with rising temperature. When the force reaches the value of the maximum, the object does not elastic counteract to the force, and gliding begins. It is also considered a vibrator with positive term, containing the deflection in second power and a term, where the deflection treats in third power (Boguslawski vibrator). Exact calculations of the dependence of the force from the temperature in adiabatic process, where the entropy is maintained constant, shows that it is represented by means of a curve with a maximum, so that stretching leads to cooling till the point of maximum is reached.
\end{abstract}

\section{Keywords}

Anharmonic Vibrator, Gliding, Destruction, Adiabatic Curve

\section{Introduction}

The subdivision of forces, acting on the atoms in crystals, on harmonic and anharmonic, where the latter presents the second approximation in the expansion of crystal lattice potential energy in series relative to the atomic displacements, 
was undertaken in works of Debye [1] and Born [2]. Detailed examination of anharmonic forces action in dielectric crystals was carried out in the works of Boguslawski [3] [4] [5], Frenkel [6] [7] Braunbek [7] [8], and Brillouin [8] [9]. In the works of Boguslawski and Frenkel the anharmonic energy arises in the exponent of statistical distribution, which is expanded in power under the premise, that this energy is small and only first power can be taken into account. In some phenomena (thermal expansion) it is so, but for considering of gliding such approximation is insufficient. Also for constructing of adiabatic curve the behavior of anharmonic vibrator must be considered more precise. Braunbek integrates the dynamical equation of motion under the action of anharmonic force, and Brilluin calculates thermodynamically the influence of temperature on the elastic moduls and occurrence the phenomena of melting, when the modul turns to zero.

In the Born works [10] [11] is asserted, that all distinctions of real crystals properties from that of ideal (harmonic approximation) are caused by anharmonic forces. An approximate formula for calculation of anharmonic forces actions is proposed. The development of such calculations is presented in works [12] [13]. The kinetic theory of strength was developed in the work [14]. In the work [15] the Boguslawski model of anharmonic vibrator was applied to consideration of electric discharge of a dielectric crystal, when an electric field is applied. But when the force is of other nature, the calculations will be almost the same; only the comprehension of the phenomenon will be changed and considered as plasticity or destruction. The phenomenon of plasticity and destruction was considered in some works; here the model of anharmonic vibrator is used for describing these phenomena. The calculations are carried out according to dynamical methodic (further development of calculations that use the virial theorem), which has in solid corps an advantage thanks to the lesser number of assumptions, which can be seen from comparing of work [16] with the works of Boguslawski. It can be also marked, that in Boguslawski calculations the positive and negative crystal directions are not equivalent, what is essential for arising of thermal expansion and pyroelectricity, but for description of transition to gliding motion the both directions of axis may be equivalent. In presented work the potential energy of the atom contains second and fourth powers of coordinate. For calculation of vibrator behavior dynamical procedure is used [15] [16]. Another example of the action of anharmonic forces is adiabatic stretching or compression, which causes change of temperature. Works in this direction raised long ago [17] [18] [19] [20], but in crystals these effects remained feebly expressed. Modern investigations use high pressures, created also by shock waves, generated in nuclear explosions [21]. In such conditions the compression is considerable, and structures conversions in crystals can be observed. Approximately the process can be considered as adiabatic, therefore to investigation of adiabatic compression of condensed corps some works are devoted [22]-[28]. The equation of adiabatic process for Boguslawski vibrator is deduced in this 
paper.

\section{Dynamical Consideration of Transition to Glide}

A symmetrical in relation to both directions of coordinate axis of molecular forces anharmonic vibrator, that potential energy as a function of coordinate is

$$
U(x)=\frac{c}{2} x^{2^{\prime}}+\frac{b}{4} x^{4}-f x ; c>0, b<0
$$

( $f$-applied force), will be considered. The graph of $U^{0}(x)$, ( $f$ is absent) is presented on Figure 1.

The coordinates of maxima have the values

$$
x= \pm \sqrt{-\frac{c}{b}} ; U^{0}=-\frac{c^{2}}{4 b}
$$

The free energy as a function of mean displacement $s$ and root mean square amplitude $u$ is presented in the form [15]

$$
F=\frac{c}{2}\left(s^{2}+u^{2}\right)+\frac{b}{4}\left(s^{4}+6 s^{2} u^{2}+3 u^{4}\right)-k T \ln u
$$

Condition of minimum in relation to $s, u$ leads to equations

$$
\begin{aligned}
& s\left(c+b s^{2}+3 b u^{2}\right)=f \\
& c+3 b s^{2}+3 b u^{2}=\frac{k T}{u^{2}}
\end{aligned}
$$

If the thermal movement is absent $u=0$, the dependence of the applied force from the displacement is represented through the equation

$$
f=c s+b s^{3}
$$

The extreme points have the coordinates

$$
s= \pm \sqrt{-\frac{c}{3 b}} ; \quad f= \pm \frac{2}{3} c \sqrt{-\frac{c}{3 b}}
$$

The stability part of the curve is placed between extreme points. When the applied force is absent, consequently $s=0$, the dependence of temperature from amplitude represents the expression

$$
k T=c u^{2}+3 b u^{4}
$$

The coordinates of maximum have the values

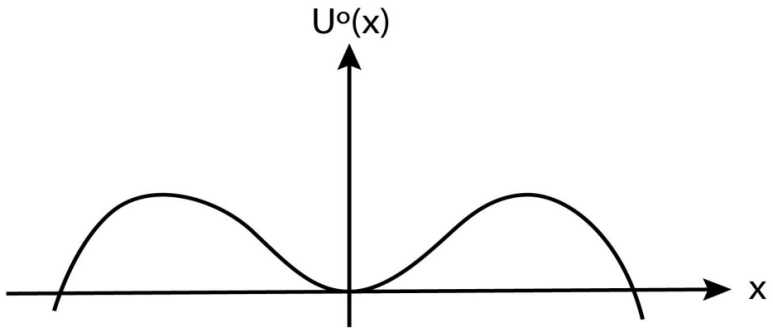

Figure 1. Graph of vibrator potential energy. 


$$
u^{2}=-\frac{c}{6 b} ; \quad k T=-\frac{c^{2}}{12 b}
$$

The states are stabile in the range from zero to abscissa of maximum amplitude. By acting of the force the expression of amplitude through displacement with retaining quadratic terms has according (5) the form

$$
u^{2}=-\frac{1}{6 b}\left[c-\sqrt{c^{2}+12 b k T}-3 b \frac{c-\sqrt{c^{2}+12 b k T}}{\sqrt{c^{2}+12 b k T}} s^{2}\right]
$$

It can be introduced the marking

$$
\tau=\sqrt{1+\frac{12 b k T}{c^{2}}}
$$

Equations (4), (5) receive the form

$$
\begin{aligned}
& u^{2}=-\frac{1-\tau}{6 b} c+\frac{1-\tau}{2 \tau} s^{2} \\
& f=\frac{c}{2}(1+\tau) s+\frac{3-\tau}{2 \tau} b s^{3}
\end{aligned}
$$

The dependence of force from the displacement for a number of temperatures is presented on Figure 2

The coordinates of force maximum have the values

$$
S_{M}=\sqrt{-\frac{\tau(1+\tau) c}{3(3-\tau) b}} ; \quad f_{M}=\frac{1}{3}(1+\tau) c S_{M}
$$

For the maximum of the amplitude will be found the expression

$$
u_{M}^{2}=-\frac{2}{3} \frac{1-\tau}{3-\tau} \cdot \frac{c}{b}
$$

which increases with rising of temperature and at temperature (9) reaches maximal value.

By rising of temperature $\tau$ aspires to zero, but, as can be seen from (12), the coefficient of expansion turns to infinity, so the solution of Equations (4), (5) must be found exact. The exact expression of amplitude is

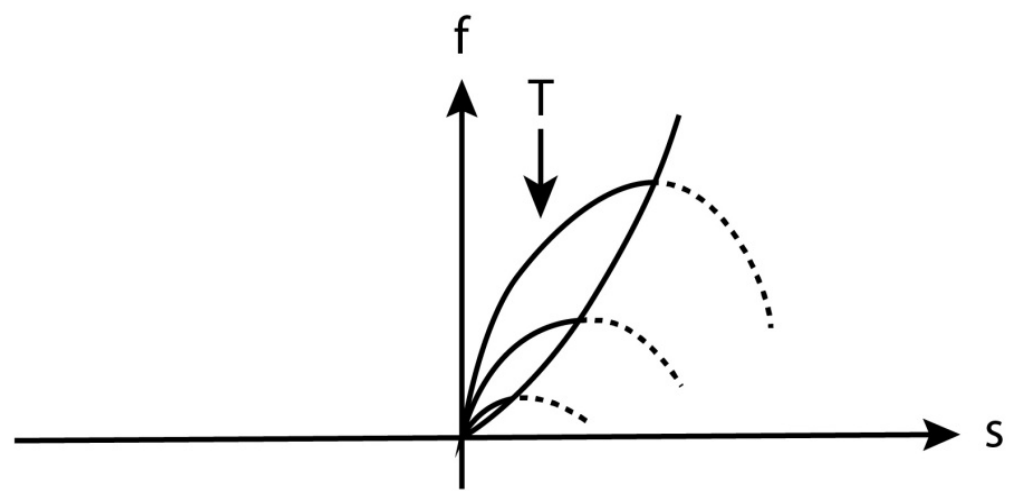

Figure 2. Dependence of force from displacement for a number of temperatures. 


$$
u^{2}=-\frac{1}{6 b}\left[c+3 b s^{2}-\sqrt{\left(c+3 b s^{2}\right)^{2}+12 b k T}\right]
$$

If we put here for the temperature the value (9), corresponding $\tau=0$, the radical will be imaginary for values of displacement different from zero, so this value will be the single solution with $f=0$ (great values of displacement, which can change this conclusion, as is evident from Figure 2, are excluded). Correct limiting value of amplitude is given trough the first term of (12), when we put $\tau=0$. Because the calculation is approximate, the conditions of stability are not formulated. Usually, the point of extremity divides the curve in stability and not stability parts. When the force overcomes the maximal value (14), the vibrator begins to glide.

\section{The Equation of Adiabatic Process}

We deduce the equation of adiabatic curve, using the results of Boguslawski vibrator calculations [15] [16]. The inner energy as a sum of kinetic and potential energies or their mean values

$$
E=W+U=\bar{W}+\bar{U}
$$

for Boguslawski vibrator with stretching force $f$ has the form

$$
E=\frac{k T}{2}+\frac{c}{2}\left(s^{2}+u^{2}\right)+\frac{a}{3}\left(s^{3}+3 s u^{2}\right)-f s
$$

His entropy is presented as follows

$$
S=\frac{k}{2} \ln \left(k T u^{2}\right)
$$

Entropy is constant, so one may put

$$
k T u^{2}=A
$$

and regard $A$ as a positive constant, depending from the quantity of entropy. The temperature as a function of amplitude is introduced in (18), and the condition of minimum energy leads to equations

$$
\begin{aligned}
& \frac{\partial E}{\partial s}=c s+a s^{2}+a u^{2}-f=0 \\
& \frac{\partial E}{\partial u}=-\frac{A}{u^{3}}+c u+2 a s u=0
\end{aligned}
$$

The conditions of stability are the following

$$
\begin{gathered}
\frac{\partial^{2} E}{\partial s^{2}}=c+2 a s>0 \\
\frac{\partial^{2} E}{\partial u^{2}}=\frac{3 A}{u^{4}}+c+2 a s>0 \\
\frac{\partial^{2} E}{\partial s^{2}} \frac{\partial^{2} E}{\partial u^{2}}-\left(\frac{\partial^{2} E}{\partial s \partial u}\right)^{2}=(c+2 a s)^{2}+(c+2 a s) \frac{3 A}{u^{4}}-4 a^{2} u^{2}>0
\end{gathered}
$$

From (22) the amplitude will be obtained 


$$
u^{2}=\sqrt{\frac{A}{c+2 a s}}
$$

and from (20), (22) the temperature

$$
k T=\sqrt{A(c+2 a s)}
$$

From (21) and (26) follows the expression for the applied force

$$
f=c s+a s^{2}+\sqrt{\frac{A}{c+2 a s}}
$$

This dependence is represented in Figure 3

From the condition of maximum

$$
\frac{\partial f}{\partial s}=c+2 a s-\frac{a^{2} \sqrt{A}}{(c+2 a s)^{\frac{3}{2}}}=0
$$

follows the values of corresponding coordinates

$$
\begin{gathered}
s_{m}=-\frac{1}{2 a}\left(c-a^{\frac{4}{5}} A^{\frac{1}{5}}\right) \\
f_{m}=-\frac{c^{2}}{4 a}+\frac{5}{4} a^{\frac{3}{5}} A^{\frac{2}{5}}
\end{gathered}
$$

The conditions of stability (23), (24) are satisfied in this point, in condition (25) the value (26) of amplitude will be substituted, then this inequality transforms to following

$$
(c+2 a s)^{5}>a^{4} A
$$

So, the point of maximum is the limiting point of stability, which is realized for smaller values of displacement. Excluding displacement from (27), (29), we receive connection between force and temperature

$$
f=-\frac{c^{2}}{4 a}+\frac{(k T)^{4}}{4 a A^{2}}+\frac{a A}{k T}
$$

The graph of this connection is represented in Figure 4.

The coordinates of the curve top are

$$
k T_{M}=a^{\frac{2}{5}} A^{\frac{3}{5}} ; \quad f_{M}=f_{m}
$$

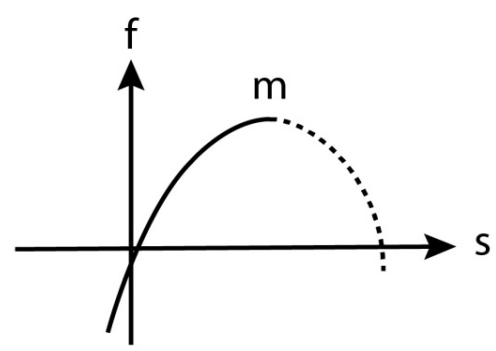

Figure 3. Dependence of force from displacement. 


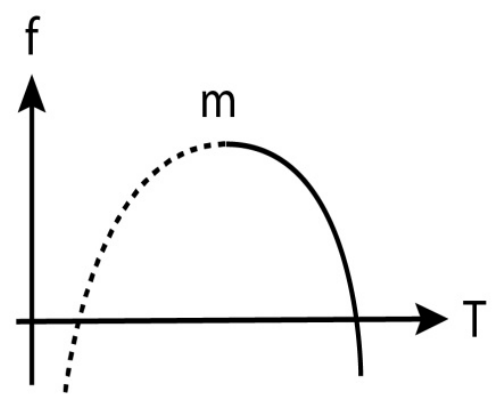

Figure 4. Dependence between force and temperature.

The stability states are placed to right side of the top, because diminishing of displacement corresponds to increase of temperature. So, the Equation (33) represents the adiabatic curve.

\section{Concluding Remarks}

Considering of anharmonic vibrator simple models gave possibility to receive the expression for the force that corresponds to the limit of elastic behavior and beginning of gliding. It is calculated the dependence of crystal temperature from the applied force in the condition of thermoisolation. More complicated models can give possibility to examine some other properties of the object. In paper [15] is considered the model of vibrator with the potential energy, which includes the coordinate in second, third and fourth powers. The graph of energy shows two differences in height maxima. It is evident, that overcoming of the low maximum leads to the lost of elastic properties, and overcoming the high to destruction. The calculation of these phenomena can be carried out, when the term in third power is small and can be neglected in zero approximation. In the first approximation when the applied force is absent the phenomenon of pyroelectricity can be taken into account. It can be of interest the calculating of gliding phenomena considering real structure of crystals with real forces of interactions between atoms, as was undertaken in [6], but with use of dynamical calculations. This is a heavy task and it is worthwhile to divide them in steps that can be considered one after another. The behavior of vibrators with more complicated dependence of potential energy from deflection can also be considered.

\section{References}

[1] Debye, P. (1915) Equation of State and Quantum Potentials with Supplement to Thermoconductivity. Report, Kinetically Theory of Matter and Electricity. Leipzig and Berlin.

[2] Born, M. (1926) Problems of Atom Dynamics. Julius Springer, Berlin. https://doi.org/10.1007/978-3-642-99600-9

[3] Boguslawski, S. (1914) Theory of Dielectrics. Dependence of Dielectric Constant from the Temperature, Pyroelectricity. Physics Journal, 15, 283-288.

[4] Boguslawski, S. (1915) About the Structure of Dielectric Crystals. Journal of Russian 
Physics-Chemical Society. Department of Physics, 47, 247-275.

[5] Boguslawski, S. (1961) Selected Works in Physics. In: Sementchenko, W.K., Ed., Pushpa Publishing House of Physics and Mathematics, Literature, Moscow,

[6] Frenkel, J. (1926) The Theory of Elasticity and Strength of Crystalline Corps. Physical Magazine, 37, 572-609.

[7] Braunbek, W. (1926) The Lattice Dynamics of Melting Process. Physical Magazine, 38, 549-572.

[8] Brilloin, L. (1938) The Thermal Dependence of Elasticity in Solids. Physical Review, 54, 916-917. https://doi.org/10.1103/PhysRev.54.916

[9] Brillouin, L. (1940) Influence of Temperature on Elasticity of Solid Corps. Notebook of Mathematical Sciences, 99. Gauthier Villars, Paris.

[10] Born, M. (1951) The Limit of Ideal Crystal Theory Applicability and Their Overcoming. Ceremonial Assay to the Celebration of Two Hundred Years Creation Academy of Sciences in Göttingen, Springer publishing, Berlin.

[11] Born, M. (1952) The New Point of View on the Theory of Condensed Matter. Reports of the Annual Meeting, Carried Out Conjointly with Commission of Thermodynamic of Physics International Union, Paris, 2-7 June 1952, 334-337.

[12] Hooton, D. (1955) A New Treatment of Anharmonicity in Lattice Thermodinamics. Philosophical Magazine, 46, 422-432. https://doi.org/10.1080/14786440408520575

[13] Hooton, D. (1955) A New Treatment of Anharmonicity in Lattice Thermodynamics. Philosophical Magazine, 46, 433-442. https://doi.org/10.1080/14786440408520576

[14] Regel, W., Slutsker, A. and Tomashewskiy, E. (1972) The Kinetically Nature of Solid Corps Strength. Progress in Physical Sciences, 106, 103-228.

[15] Kozlovskiy, V.Kh. (1967) Dynamical Theory of Pyroelectric Vibrators. News of Academy of Sciences USSR, 31, 1054-1059.

[16] Kozlovskiy, V.Kh. (2016) The Boguslawski Melting Model. World Journal of Condensed Matter Physics, 6, 45-55. https://doi.org/10.4236/wjcmp.2016.61007

[17] Thomson, W. (1853) On the Dynamical Theory of Heat. Transactions of the Royal Society of Edinburgh, 20, 261-298. https://doi.org/10.1017/S0080456800033172

[18] Kelvin, L. (2011) Elasticity and Heat. Mathematical and Physical Papers, 3, 236-239.

[19] Joule, J.P. (1857) On the Thermoelasticity of Ferruginous Metals and on Thermal Effects of Stretching Solid Bodies. Proceedings of the Royal Society, London, 8, 355-356. https://doi.org/10.1098/rspl.1856.0093

[20] Joule, J.P. (1857) On the Thermal Effect of Longitudinal Compression of Solids. Proceedings of the Royal Society, London, 8, 564-566. https://doi.org/10.1098/rspl.1856.0147

[21] Trunin, R. (1994) The Shock Compressibility of Condensed Matter in Powerful Shock Waves of Underground Nuclear Explosions. Progress in Physical Sciences, 164, 1214-1237.

[22] Slutsker, A.I. and Volodin, V.P. (1994) Microscopic of the Thermoelastic Effect. Thermochimica Acta, 247, 111-119. https://doi.org/10.1016/0040-6031(94)80116-9

[23] Volodin, V.P. and Slutsker, A.I. (1994) Specific Features of the Thermoelastic Effect in Polymers. Thermochimica Acta, 247, 121-128. https://doi.org/10.1016/0040-6031(94)80117-7

[24] Giljarow, W.L., Slutsker, A.I., Volodin, V.P. and Laius, L.A. (1997) The Energetic of Adiabatically Loaded Exited Anharmonic Vibrator. Physics of Solid Corps, 39, 153- 
157.

[25] Giljarow, W.L., Slutsker, A.I., Volodin, V.P. and Laius, L.A. (1998) The Energetic of Thermoelasticity Effect in Solid Corps. Physics of Solid Corps, 40, 1548-1551.

[26] Slutsker, A.I., Giljarow, W.L. and Lukjanenko, A.S. (2006) Energetic Peculiarities of Adiabatically Loaded Anharmonic Vibrator. Physics of Solid Corps, 48, 1832-1837.

[27] Slutsker, A.I., Polykarpow, Ju.I., Karow, D.D. and Hoffmann, I.V. (2013) Energetics of Elastically Loaded Anharmonic Solid Corps. Physics of Solid Corps, 55, 510-516.

[28] Gorobey, N.N. and Lukjanenko, A.S. (2015) About the Mechanical Equilibrium of Warmed Anharmonic Solid Corps. Physics of Solid Corps, 57, 91-94.

Submit or recommend next manuscript to SCIRP and we will provide best service for you:

Accepting pre-submission inquiries through Email, Facebook, LinkedIn, Twitter, etc. A wide selection of journals (inclusive of 9 subjects, more than 200 journals)

Providing 24-hour high-quality service

User-friendly online submission system

Fair and swift peer-review system

Efficient typesetting and proofreading procedure

Display of the result of downloads and visits, as well as the number of cited articles

Maximum dissemination of your research work

Submit your manuscript at: http://papersubmission.scirp.org/

Or contactwjcmp@scirp.org 